\title{
Uncovering the mechanisms of research capacity development in health and social care: a realist synthesis
}

\author{
Jo Cooke ${ }^{1}$, Paolo Gardois ${ }^{2}$ and Andrew Booth ${ }^{3^{*}}$ (D)
}

\begin{abstract}
Background: Research capacity development (RCD) is considered fundamental to closing the evidence-practice gap, thereby contributing to health, wealth and knowledge for practice. Numerous frameworks and models have been proposed for RCD, but there is little evidence of what works for whom and under what circumstances. There is a need to identify mechanisms by which candidate interventions or clusters of interventions might achieve RCD and contribute to societal impact, thereby proving meaningful to stakeholders.

Methods: A realist synthesis was used to develop programme theories for RCD. Structured database searches were conducted across seven databases to identify papers examining RCD in a health or social care context (1998-2013). In addition, citation searches for 10 key articles (citation pearls) were conducted across Google Scholar and Web of Science. Of 214 included articles, 116 reported on specific interventions or initiatives or their evaluation. The remaining 98 articles were discussion papers or explicitly sought to make a theoretical contribution. A core set of 36 RCD theoretical and conceptual papers were selected and analysed to generate mechanisms that map across macro contexts (individual, team, organisational, network). Data were extracted by means of 'If-Then' statements into an Excel spreadsheet. Models and frameworks were deconstructed into their original elements.
\end{abstract}

Results: Eight overarching programme theories were identified featuring mechanisms that were triggered across multiple contexts. Three of these fulfilled a symbolic role in signalling the importance of RCD (e.g. positive role models, signal importance, make a difference), whilst the remainder were more functional (e.g. liberate talents, release resource, exceed sum of parts, learning by doing and co-production of knowledge). Outcomes from one mechanism produced changes in context to stimulate mechanisms in other activities. The eight programme theories were validated with findings from 10 systematic reviews (2014-2017).

Conclusions: This realist synthesis is the starting point for constructing an RCD framework shaped by these programme theories. Future work is required to further test and refine these findings against empirical data from intervention studies.

Keywords: Research capacity development, Realist synthesis, Evaluation, Leadership, Training

\footnotetext{
* Correspondence: a.booth@sheffield.ac.uk

${ }^{3}$ School of Health and Related Research, University of Sheffield, Regent Court,

30 Regent Street, Sheffield S1 4DA, United Kingdom

Full list of author information is available at the end of the article
}

(c) The Author(s). 2018 Open Access This article is distributed under the terms of the Creative Commons Attribution 4.0 International License (http://creativecommons.org/licenses/by/4.0/), which permits unrestricted use, distribution, and reproduction in any medium, provided you give appropriate credit to the original author(s) and the source, provide a link to the Creative Commons license, and indicate if changes were made. The Creative Commons Public Domain Dedication waiver (http://creativecommons.org/publicdomain/zero/1.0/) applies to the data made available in this article, unless otherwise stated. 


\section{Background}

National policy and financial investment across the globe indicates overwhelming support for building research capacity in healthcare systems. Enhanced capacity is believed to promote problem solving [1], reduce the gap between evidence and practice [2, 3], and promote health gains [4]. It is considered a powerful and cost-effective way of advancing healthcare and development [5] and, if done well, can improve collaboration between high- and low-income countries [1] and address health inequalities [6]. United Kingdom policy highlights that the 'best' health research promotes the health and wealth of the nation [7].

Despite overwhelming support in the research literature and policy documents, research capacity development (RCD) is poorly defined, and conceptually elusive [8]. Undertaking a conceptual review of the literature, Condell and Begley define research capacity-building, a component of RCD, as "a funded, dynamic intervention operationalised through a range of foci and levels to augment ability to carry out research or achieve objectives in the field of research over the long-term, with aspects of social change as an ultimate outcome" [8]. This definition highlights that RCD is complex and operates at a number of structural levels, including individual, team, organisational and within networks [9], and includes a range of 'interventions' [10] or foci of activity. RCD activities are often conducted in parallel and can be interrelated. Research training, fellowships and mentorship schemes, for example, can be planned and evaluated separately, but in practice are often linked [11] and add value to each other. RCD 'interventions' may include processes such as priority-setting [12], but equally can incorporate structural changes in organisations, for example, developing an information technology infrastructure $[13,14]$. Structural and process interventions can link to outcomes of their own but can also produce a fertile environment for other RCD initiatives. Collectively and separately, they achieve the common goal of stimulating 'more research done well'.

The challenge of understanding RCD and how it operates is compounded by the fact that RCD is often seen as a means to an end [9] rather than an end in itself. From this perspective, means are the skills and structural enhancements that enable research to be conducted with the ultimate 'end' or goal of RCD in healthcare being to change practice and systems to improve health [13]. Setting the criteria for impact to this level makes it even more challenging to understand 'what works' in RCD. Although targeting improved health as the goal gives more value and 'meaning' to RCD activity, it necessitates long and convoluted causal chains, making it even more difficult to attribute impact. It also requires consideration of the nature and quality of how RCD interventions are performed, whether they are 'meaningful' and whether they can produce change in practice. Nuyens, for example, suggests that how priority-setting is undertaken influences how meaningful it is, and whether research is subsequently used in practice [15].

The literature on RCD reflects its elusive and chameleon-like quality and how difficult it is to measure and attribute impact [16]. Reports and case studies of RCD interventions in health do exist but evidence on effectiveness is inconclusive. Many evaluations fall short of being able to determine the impact on healthcare systems and thus demonstrate meaningful RCD. Such limitations may, in turn, reflect shortcomings in the theoretical underpinning of RCD interventions [16].

Further complexity is added when considering the relationship between RCD and knowledge mobilisation (KM). RCD focuses very much on empowering and enabling different levels of the health research system to conduct research. KM focuses further downstream once the capacity is in place, to what extent can the research that has been generated change practice? Many health systems display an evolutionary or developmental aspect to these activities; in the United Kingdom, for example, antecedent investment in research and development support units has started to deliver the capacity to mobilise knowledge through networks such as the Collaborations for Leadership in Applied Health Research and Care (CLAHRCs). However, typically, RCD and KM co-exist and interact given that new requirements identified from KM will feed into subsequent requirements for specific reconfigurations of research capacity.

In summary, RCD can be categorised as a complex intervention, what Willis et al. describe as " $a$ set of purposefully coordinated components that target multiple levels and sectors of a system, that operate both independently and inter-dependently, and that interact in the contexts in which they are implemented" [17]. Realist approaches offer an ideal methodology for understanding, evaluating and planning such interventions.

Numerous models suggest how RCD works [1, 9, 14, 18-21]. However, few models address underlying mechanisms for what works, and why, across a range of contexts, in achieving a meaningful impact on health services and systems. No attempt has been undertaken to link existing RCD models, nor to develop theories in a systematic way from them.

The objective of this realist synthesis is to address what are the mechanisms that support meaningful RCD that are triggered across diverse contexts, specifically at individual, team, organisation or network level, as described in conceptual and theoretical papers? We aim to isolate mechanisms that are activated across and within diverse contexts in order to develop programme theories that will identify and test causal chains in RCD programmes. 


\section{Methods}

This paper reports on a work package within a larger programme of work on reviewing the RCD literature in health and social care. The wider programme aims to analyse both the conceptual papers and intervention studies. This paper reports on the first stage of this research, namely a realist synthesis of theoretical and conceptual papers to develop programme theory at a macro level. A realist synthesis seeks to explain and unpack the mechanisms by which an intervention works (or fails to work); it seeks to advance a potential explanation, as opposed to a definitive judgement, about how interventions (in this case research capacity development activities) achieve their outcomes [22]. By mechanisms we mean the responses that the 'active' components within an intervention stimulate, either individually or collectively, within participants. We explore these mechanisms by first identifying an accessible explanation of how the intervention is understood to work, known as a 'programme theory', derived from the research literature, official documents or stakeholder explanations.

A realist approach recognises that the context within which an intervention is delivered is "complex, multi-faceted and dynamic" [23]. It challenges the assumption, implicit in conventional systematic review methodology, that the same intervention will work in the same way in different contexts. Realist logic seeks to articulate statements along the lines of 'IF Context A includes... THEN Mechanisms X, Y, Z are activated LEADING TO Outcome $\mathrm{O}^{1}$ - these statements are technically known as 'CMO Configurations' or 'CMO chains'.

The work described in this study seeks to identify mechanisms that are activated across a variety of contexts operating at different levels (individual, team, organisational, network) within the health research system to achieve either desirable or unintended outcomes. Subsequent work will seek to map evidence from empirical studies to the theoretical framework. The realist synthesis method was supported by systematic mapping methodology. A core set of theoretical and conceptual papers on RCD was analysed in order to generate mechanisms and map these across macro contexts (individual, team, organisational, network) in order to develop programme theories.

\section{Initial scoping of the literature}

In 2005, a scoping review, Re:Cap - Identifying the Evidence Base for Research Capacity Development in Health and Social Care [10], was commissioned by the National Coordinating Centre for Research Capacity Development, in partnership with the National Steering Committee of Research and Development Support Units, to "identify, map, and describe the literature available to inform research capacity development (RCD) activities in health and social care, and to inform the work of RDSUs [Research and Development Support Units]" (the then United Kingdom regional research and development support units) [10]. A scoping review is "a preliminary assessment of the potential size and scope of the available research literature" [24] and does not seek to conduct formal assessments of evidence quality. One component of the scoping review sought to identify existing RCD models, frameworks and theories. Four limitations were identified for this component of the wider scoping review, namely (1) models, frameworks and theories were derived from a heterogeneous range of sources and disciplines making comparison and specific application to health and social care problematic, especially as some models originated from outside the context of research capacity; (2) no recognised procedures existed for identifying models, frameworks and theories in a systematic way; (3) time constraints did not permit a formal attempt to examine an empirical base for each identified model, framework or theory; and (4) related to (3), there was limited opportunity to link the theories identified to eight RCD activities (Box 1) prioritised for the scoping review. Several years later, two authors of the original scoping review (JC \& $\mathrm{AB})$, working with a trained information specialist (PG), therefore sought to return to the topic area to consolidate opportunities identified from the quantity and characteristics of the literature in order to extend the conceptual thinking that underpins RCD.

A considerable body of literature describes models for RCD together with evaluations of specific individual RCD activities. However, none of the identified studies sought to explore beneath the level of actual interventions to examine the mechanisms by which interventions might achieve their intended effects. We hypothesised that some of these effects would be specific to the context of research capacity.

\section{Search strategy}

Structured database searches were conducted across seven databases (MEDLINE, EMBASE, ASSIA, CINAHL, ERIC, PsycInfo, and Web of Science) to identify papers that examined RCD in a health or social care context. Searches were conducted across the period 1998-2013. In addition, given the diversity of terminology, 10 key articles were selected from the original Re:CAP review [10] and designated as 'citation pearls'. Citation searches were then conducted across Google Scholar and Web of Science for articles citing these conceptual works.

\section{Inclusion/exclusion criteria}

For inclusion in the initial review project a paper should:

1. Describe an RCD model/theory/framework OR 
2. Evaluate a model/theory/framework cited from elsewhere OR

3. Report an evaluation of an intervention that was based on or cites model/theory/framework

\section{AND}

4. Be specific to a health or social care setting

5. Be published in English between 1999 and 2013

Note that subsequent phases of the project required validation and refreshment of the original dataset to extend coverage between 2014 and 2017. For details see below.

\section{Keywords}

The Research question was formulated according to the BeHeMoTH question structure [25]. This formulation is specifically designed to help to specify theory-related literature searches, as follows:

Be - Behaviour of Interest: RCD (including eight specific interventions: Prioritisation; Mentoring for research; Research leadership; Research facilitation; Research skills training; Funding (including bursaries and fellowships); Networks and collaborations; Infrastructure).

H - Health Context: Health and social care. E - Exclusions: Capacity development for other (non-research) purposes; Models of RCD not tried or proposed for a health and social care context.

MoTh - Models or Theories: operationalised as a generic 'model" or theor" or concept" or framework" strategy together with named models or theories if required. Illustrative keywords are given in Table 1.

\section{Key citation pearls}

A list of 10 previous models identified for the Re:CAP project $[1,6,9,14,18-21,26,27]$ (designated as 'citation pearls') was searched in 2014 using Google Scholar, Web of Science and Scopus. Supplementary search approaches proved of particular importance given the significant variation in terminology and the update role of the review. In addition, reference lists of included articles were examined for additional references not retrieved by the database keywords search. An update procedure was conducted, specifically for systematic reviews, in December 2017 (see the section Validation and refreshment of programme theories below).

\section{Date and language restrictions}

Papers were published between 2000 and 2015. Only English language papers were considered given the intended target audience for the review findings.

\section{Quality assessment}

No accepted instruments have been developed to assess the theoretical sufficiency of conceptual papers. Our overall goal was interpretative (configurative), not aggregative [28], so we did not exclude any studies based on study quality alone. However, we did examine included papers in relation to their perceived proximity to the United Kingdom context for which we were producing the review [29].

\section{Data extraction}

Data were extracted on author, year, country, context and, where appropriate, RCD activity and study type. For this work package, articles with RCD theories, models and frameworks were used to extract causative relations of components within them in the form of 'If-Then' statements. The level at which the activity was described

Table 1 Summary of search strategies and search terms

\begin{tabular}{|c|c|c|c|c|c|c|}
\hline \multicolumn{2}{|l|}{ Research } & \multicolumn{2}{|l|}{ Capacity development } & \multicolumn{2}{|l|}{ Health and social care } & \multirow{2}{*}{$\begin{array}{l}\text { Models, etc. } \\
\text { model* OR theor* OR }^{*} \text { concept* OR framework* }^{*}\end{array}$} \\
\hline Research & AND & $\begin{array}{l}\text { 'capacity development' OR 'capacity } \\
\text { building' OR 'capacity evaluation' OR } \\
\text { 'community development' OR } \\
\text { 'community building' OR 'building } \\
\text { communities' }\end{array}$ & AND & $\begin{array}{l}\text { Not specified on health/social } \\
\text { care databases }\end{array}$ & AND & \\
\hline & & $\begin{array}{l}\text { prioritis* OR prioritiz* OR mentor* OR } \\
\text { leader* OR facilitat* OR training OR OR } \\
\text { funding OR bursaries OR fellowship* } \\
\text { OR network* OR collaboration* OR } \\
\text { infrastructure* }\end{array}$ & & \multirow{2}{*}{\multicolumn{2}{|c|}{$\begin{array}{l}\text { On non-health/social care } \\
\text { databases: } \\
\text { Health OR Nurs* OR Medical } \\
\text { OR doctor OR paramedic* OR } \\
\text { therapy OR therapist OR } \\
\text { Physiotherap* OR 'social work*' }\end{array}$}} & \\
\hline & $\begin{array}{l}\text { NEAR/ SAME/ ADJ/ } \\
\text { WITH }^{\mathrm{a}}\end{array}$ & $\begin{array}{l}\text { 'capability' OR 'capacity' OR 'productivity' } \\
\text { OR 'output' OR 'strategy'. }\end{array}$ & & & & \\
\hline \multicolumn{7}{|c|}{ Research capacity } \\
\hline \multicolumn{7}{|c|}{ Researcher development } \\
\hline \multicolumn{2}{|c|}{ Researcher career* } & & & & & \\
\hline
\end{tabular}

${ }^{\mathrm{a}}$ According to Database functionality 
as taking place was also extracted (individual, team, organisation and network level). These data were extracted into Excel sheets.

\section{Data synthesis}

\section{Accessing the programme theories}

Careful reading and re-reading of identified conceptual papers was undertaken individually by two investigators (AB, JC). Particular attention was focused on identifying causal chains by which an RCD programme or specific intervention might achieve either proximal (e.g. knowledge or skill gains) or long-term gains (i.e. improvements in health, increases in wealth or the achievement of an evidence-formed organisation or society). Data were extracted as If-Then statements into an Excel spreadsheet [30].

In cases of uncertainty, the If-Then statements, and proposed causal links, were discussed within the team. Chains of If-Then statements were constructed to yield insights on overall generic RCD approaches. A further complexity related to the level at which particular activities might be targeted, for example, at an individual, team, organisation or network level. If-Then statements were grouped by level of targeted intervention, with mechanisms that occurred at more than one contextual level being identified. In line with realist approaches, the team reasoned that similarities in mechanism, as opposed to the actual activities themselves, would extend across all levels of operations (context), to enable programme theory building.

\section{Validation and refreshment of programme theories}

An essential feature of the realist synthesis method is the process of validating the original programme theories against a further independent dataset. Citation searches were repeated for the 10 citation pearls in December 2017 and all reviews published between 2014 and 2017 meeting the original inclusion criteria and exhibiting a recognisable degree of systematicity were analysed for confirmatory and original programme theory.

\section{Results}

\section{Literature base}

The literature search was conducted across nine databases. Potentially relevant articles $(n=2763)$ were identified from searching electronic databases, and 14 additional potentially relevant articles were identified from follow-up of models and framework papers included in the earlier Re:CAP scoping review (the designated 'citation pearls'). Of the potentially relevant articles, 2081 were excluded because their primary focus was not on RCD, leaving 682 potentially relevant papers from electronic databases; 468 articles were subsequently excluded at abstract stage, being either not relevant, only available in abstract form or written in a language other than English and 214 articles from electronic databases were retained, 116 of which reported on specific interventions or initiatives or their evaluation. A subset of 98 articles were discussion papers or explicitly sought to make a theoretical contribution. These 98 articles were read carefully by one of the review team (JC/AB) and, where the study was considered appropriate to the review question, selected for data extraction. A final list of 36 conceptual papers, reflecting consensus between the reviewers, were ultimately included. Figure 1 outlines the PRISMA diagram.

The 36 papers (Table 2) reflected international interest in RCD, with prominent players including the United Kingdom $(n=11)$, Australia $(n=6)$, the United States of America $(n=5)$, Canada [31] and Canada/United States combined [27] (North America). Five papers represented international perspectives, typically in the form of literature reviews. Low- and middle-income countries were represented by five collective papers and individual papers from Bangladesh [5], Liberia [20] and South Africa [21]. Primary care was the most prevalent field of research $(n=12)$, with nursing $(n=10)$, health and health services research $(n=9)$ also being well represented. Three papers examined allied health $(n=3)$ and the final paper looked at public health [21]. Collectively, the papers represented all the identified activities of RCD, with richer papers yielding programme theory relating to three or more activities (Table 2).

Given that the aim of the project was interpretative and explanatory it was not considered necessary to comprehensively sample every possible RCD model, framework or theory. Instead, we were looking to construct theory and therefore employed a purposeful sampling approach described as 'intensity sampling'. In the context of a research synthesis, intensity sampling involves selecting papers that are "excellent or rich examples of the phenomenon of interest, but not highly unusual cases... cases that manifest sufficient intensity to illuminate the nature of success or failure, but not at the extreme" [32]. This method of sampling appears particularly appropriate given that the intent of realist synthesis is to delve into inconsistencies of evidence in order to build programme theories to offer to policy-makers [29]. Sampling was operationalised through initial selection of the 10 citation pearls and, subsequently, through selection of articles that specifically sought to theorise or conceptualise RCD.

\section{Literature classification}

The final list of included studies comprised 36 theoretical and conceptual papers (Table 2). The included literature reflected a wide range of environments and 

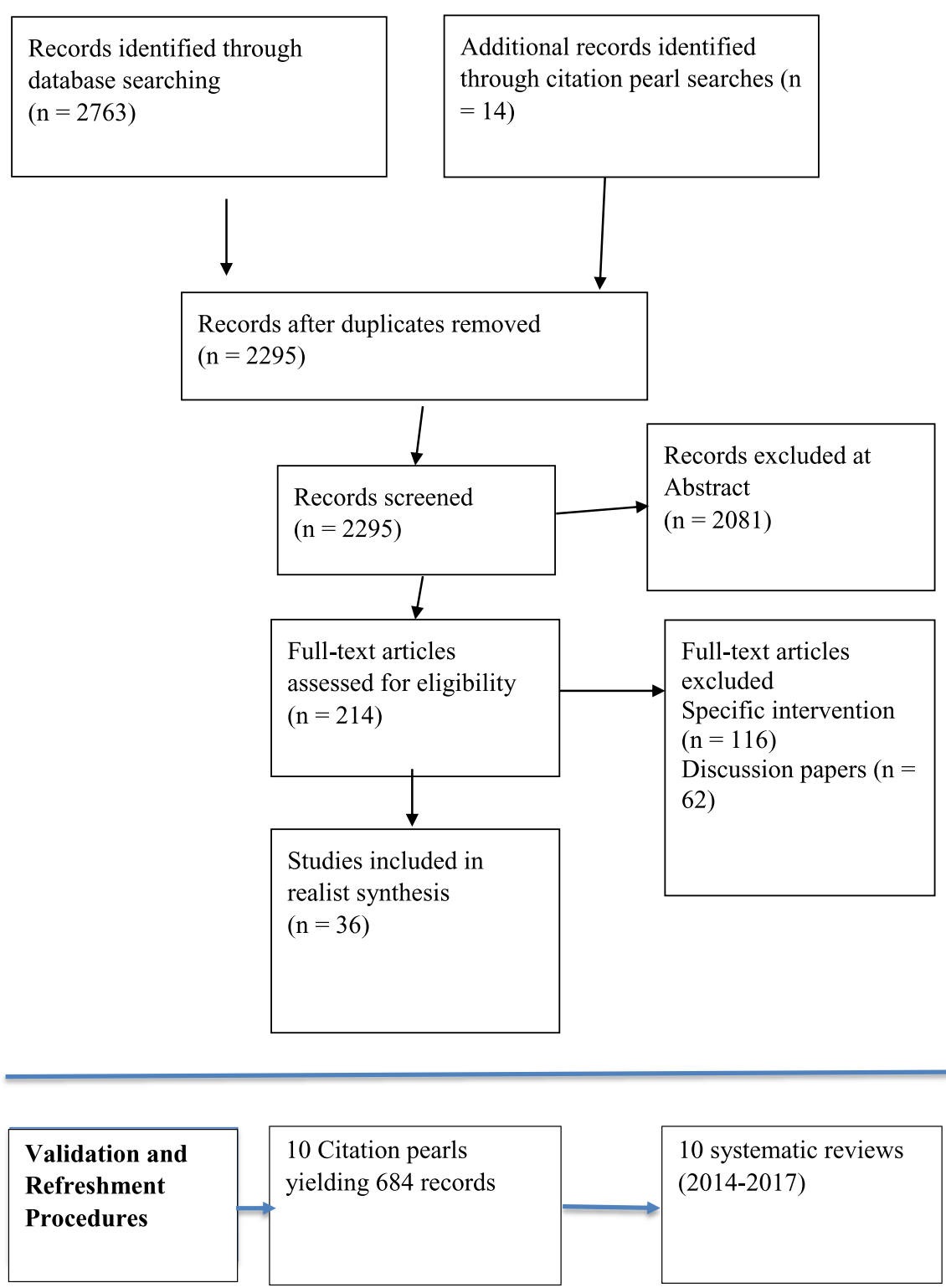

Fig. 1 Flowchart for the systematic review following the PRISMA reporting methodology

settings within which RCD might take place. The applied context for this synthesis meant we were particularly interested in United Kingdom-based studies, although Australia and the United States were particularly well represented. Primary care as a context was particularly prominent. Reasons for this may be temporal, with acute hospital infrastructures being at a later stage of development than primary care counterparts, related to saleability, with primary care organisations and networks being more able to facilitate actionable change, or may relate to external developments and priorities. We also identified papers that described North-South partnerships as exemplars of collaborations or networks.

\section{The focus of this paper}

This paper focuses on 36 conceptual and theoretical papers (Table 2), including papers containing some form of framework or model of RCD. Models and frameworks were handled in the same way and were deconstructed into their original elements (in the form of If-Then statements). Thus, hypothesised relationships were not privileged within the original deconstruction but emerged naturally from the synthesised data.

A process of discussion and consensus led to identification of eight overarching programme theories, identifying mechanisms triggered across multiple (i.e. at least more than one) macro contexts (Table 3). Several programme theories recognise RCD as a collaborative effort facilitating 


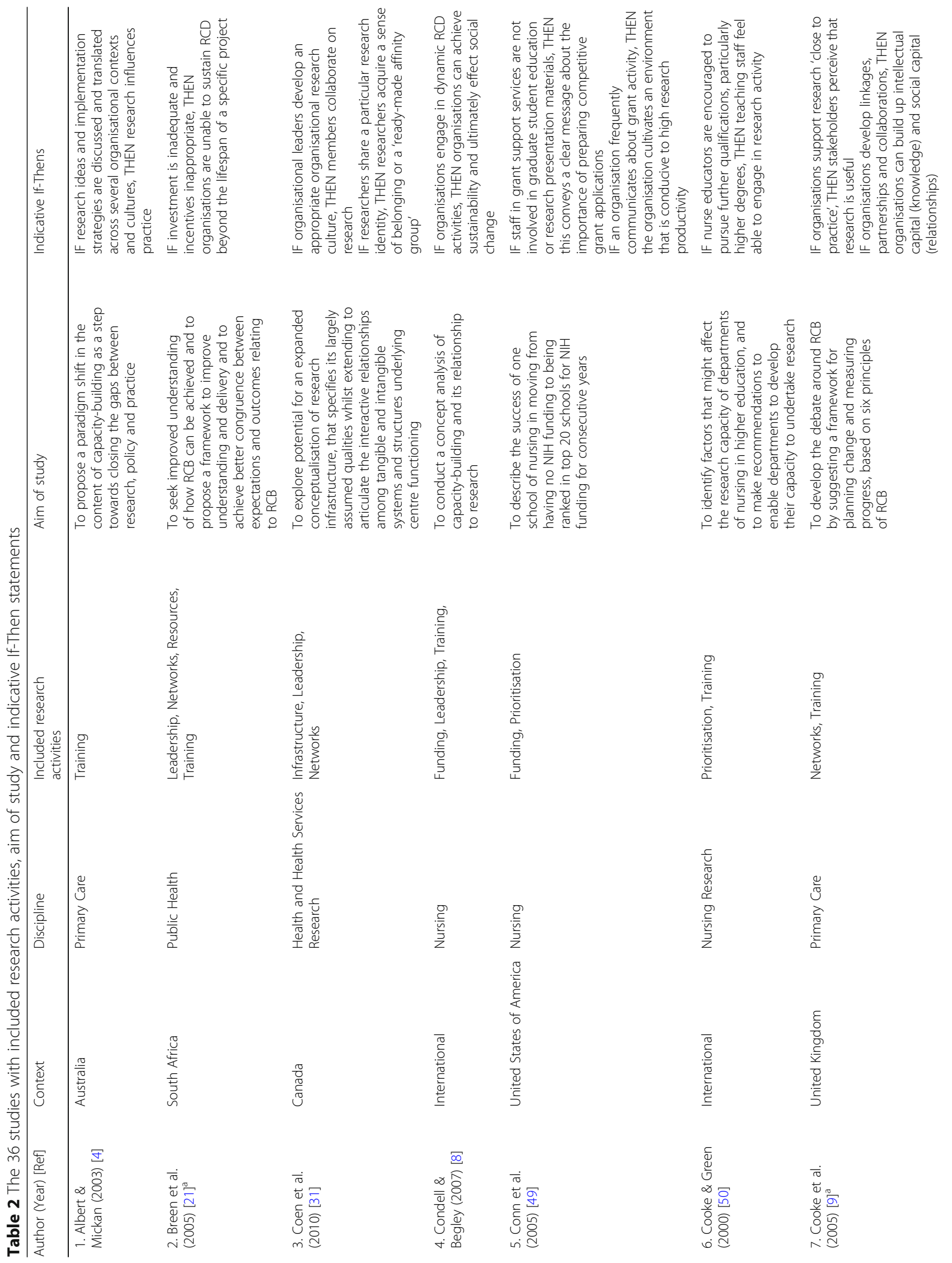




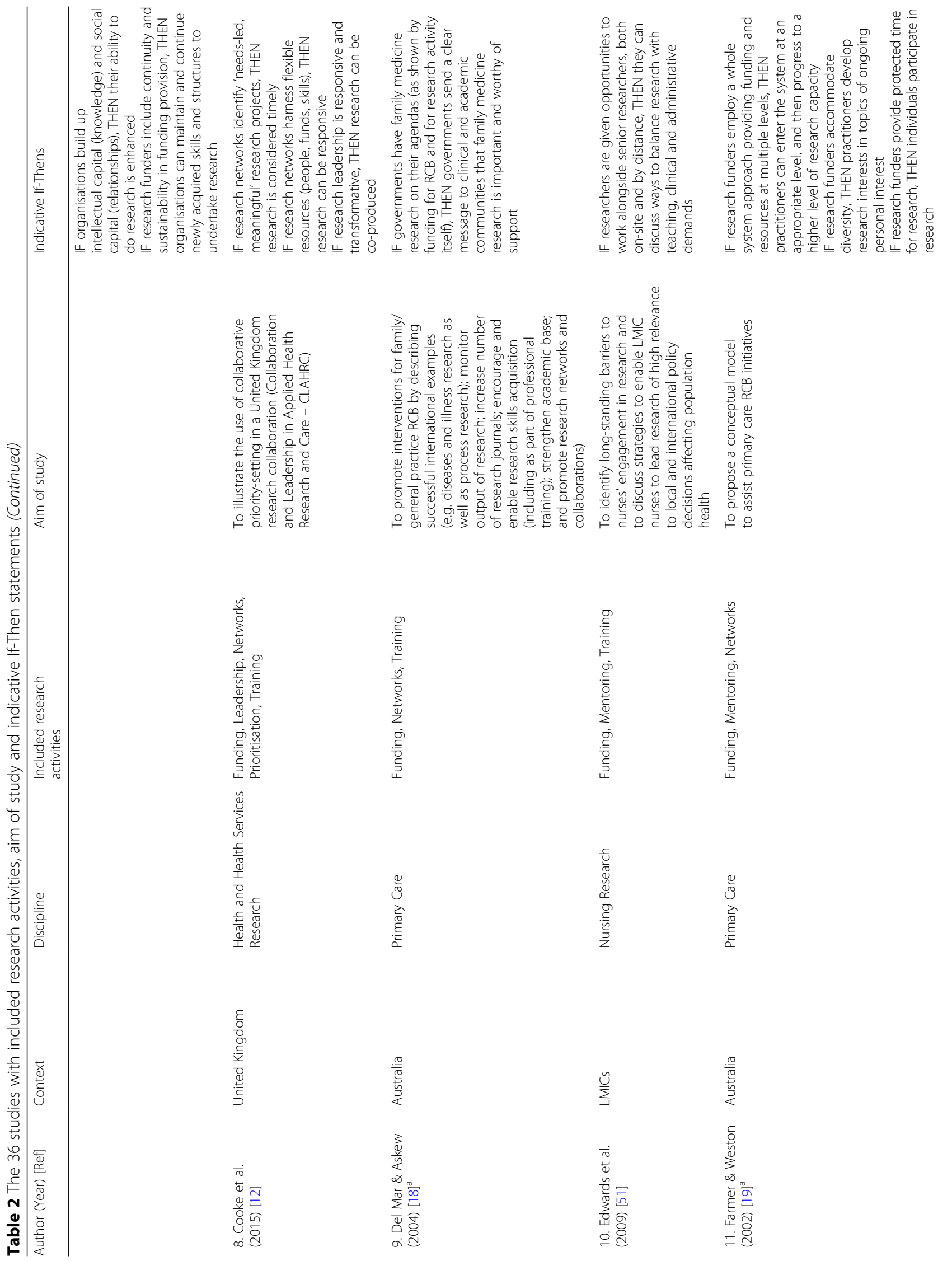




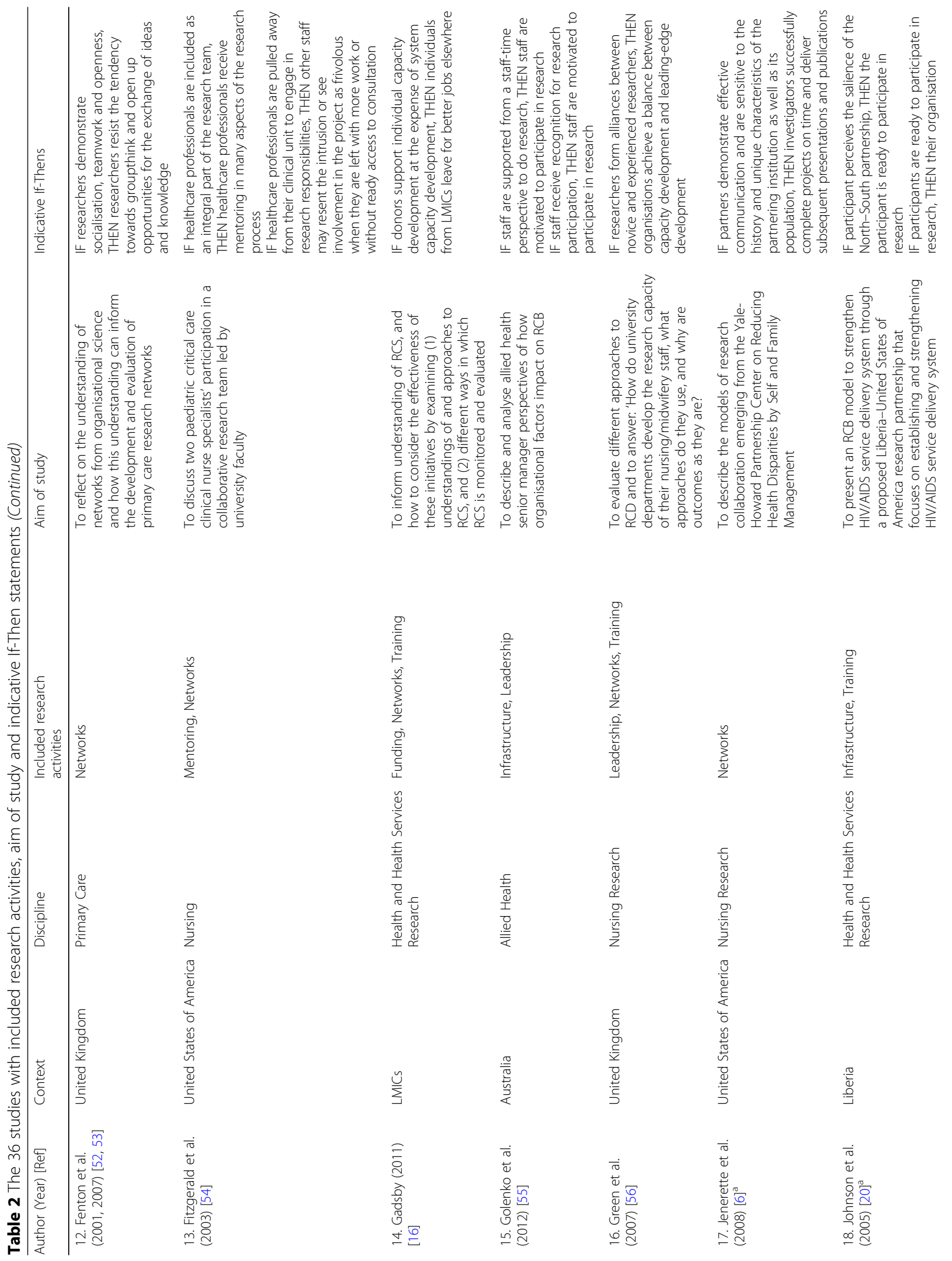




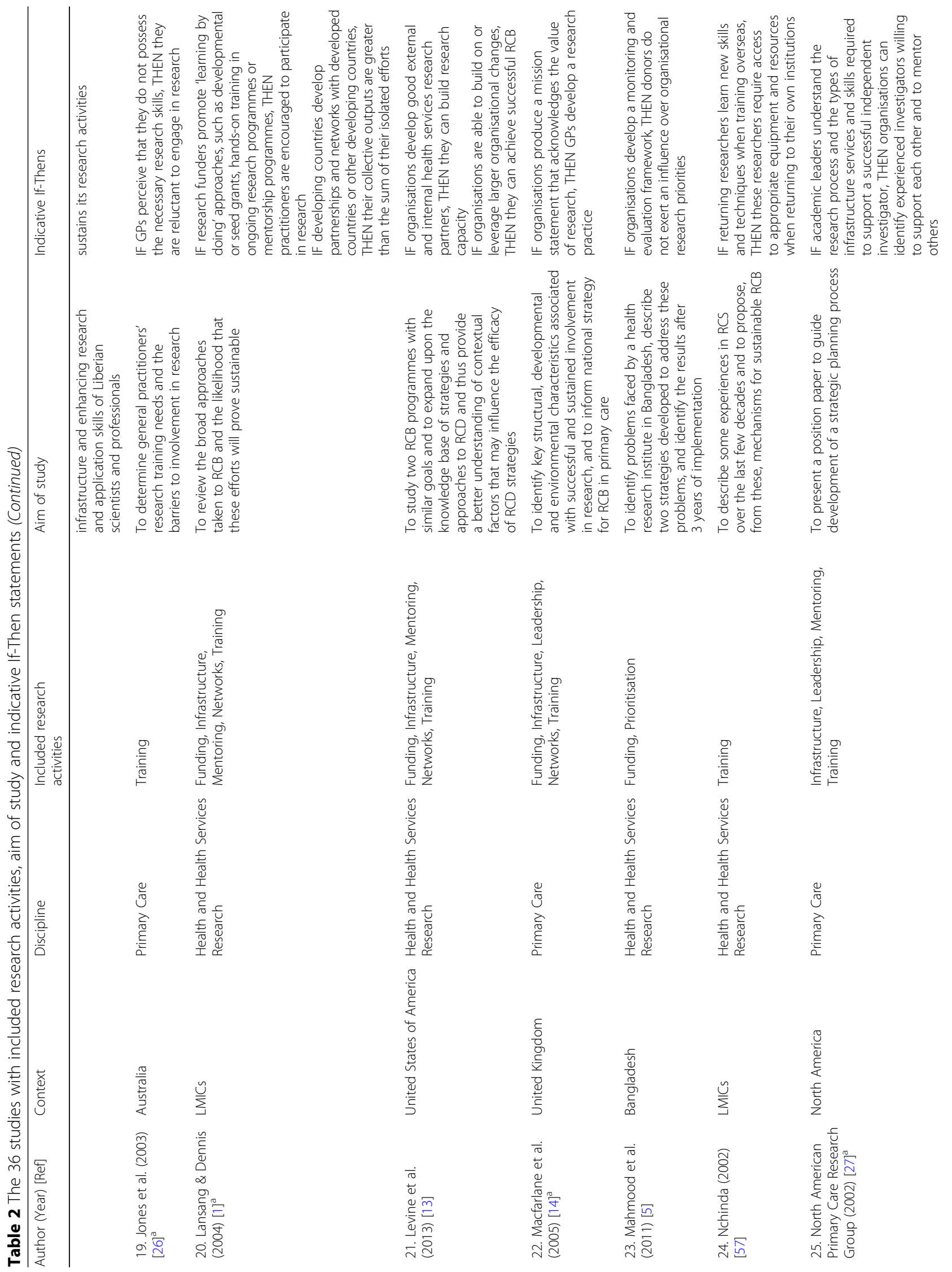




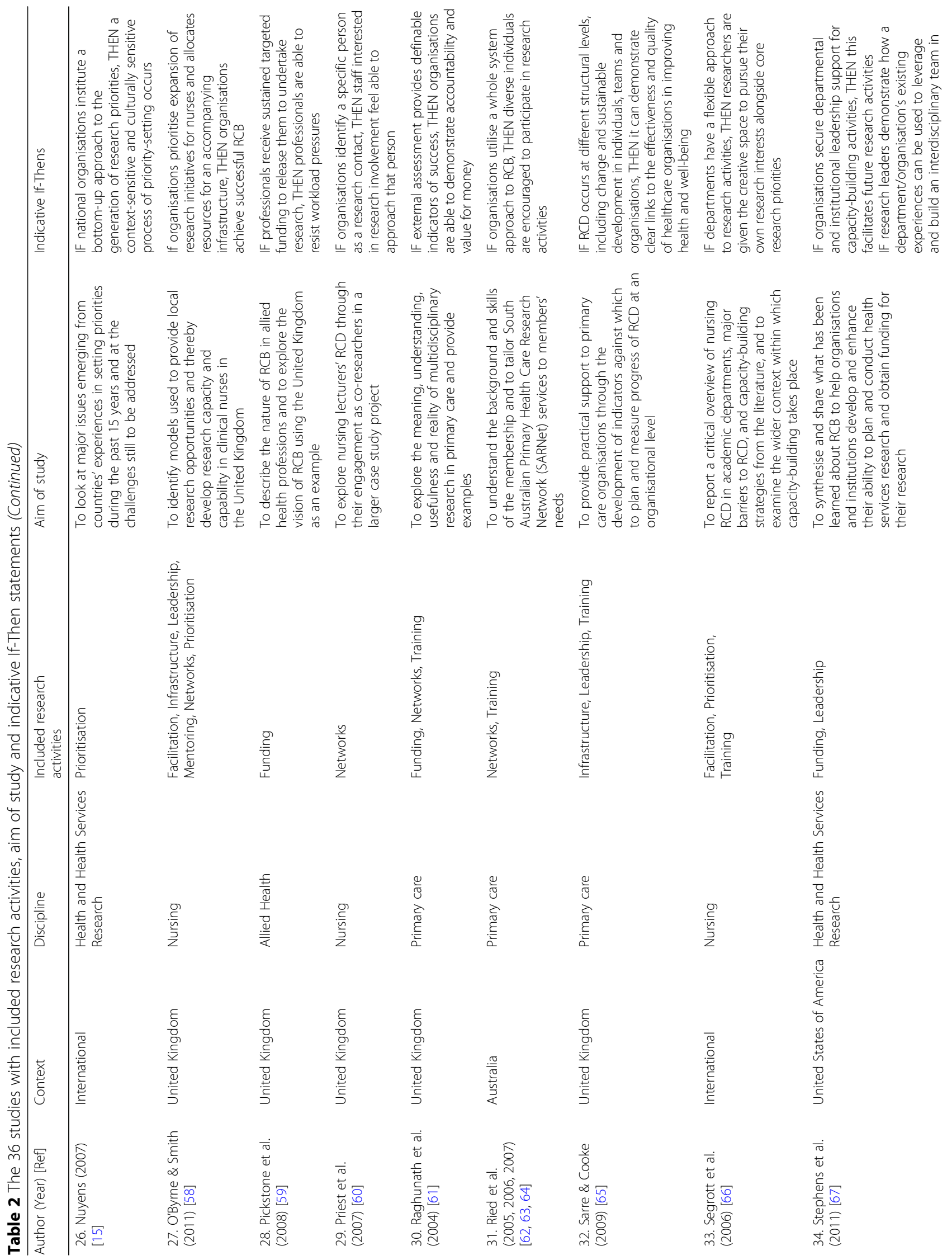




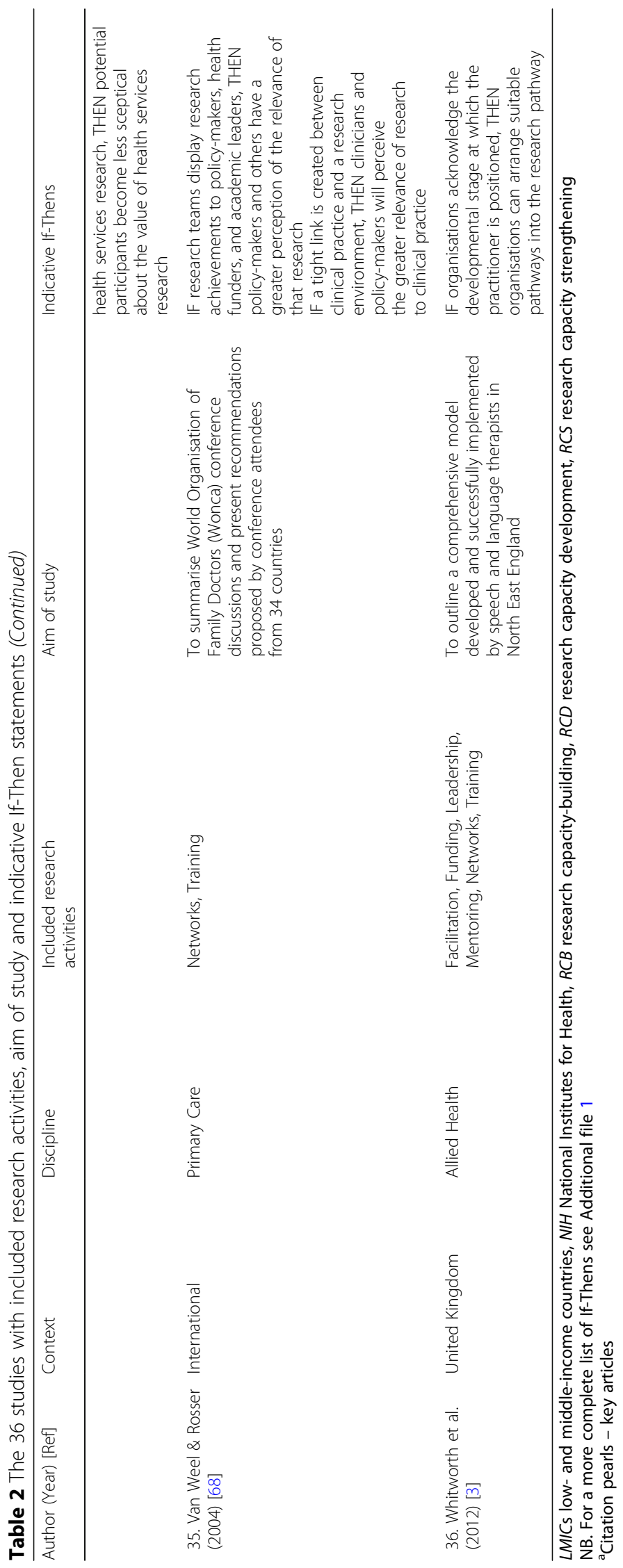




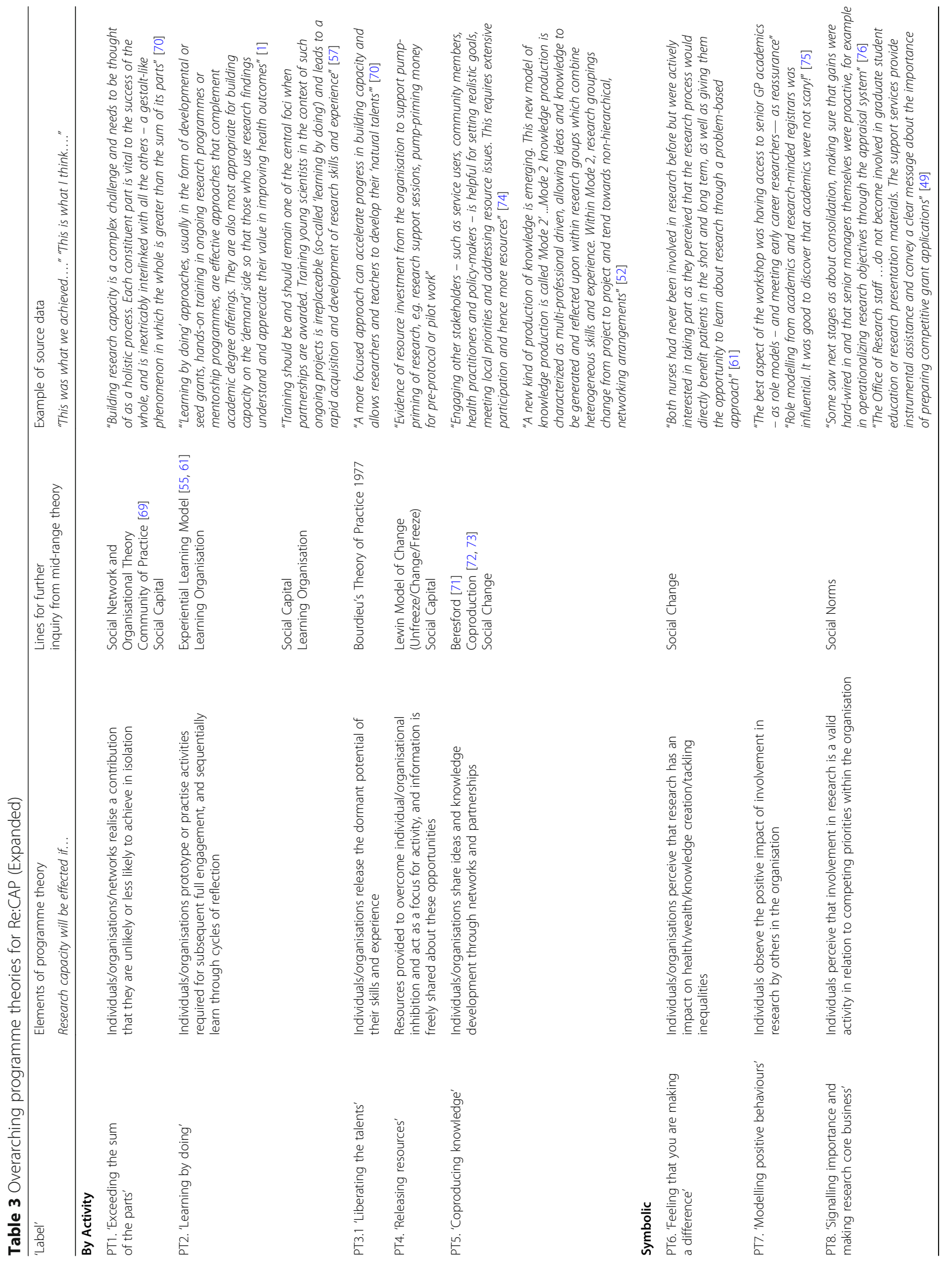




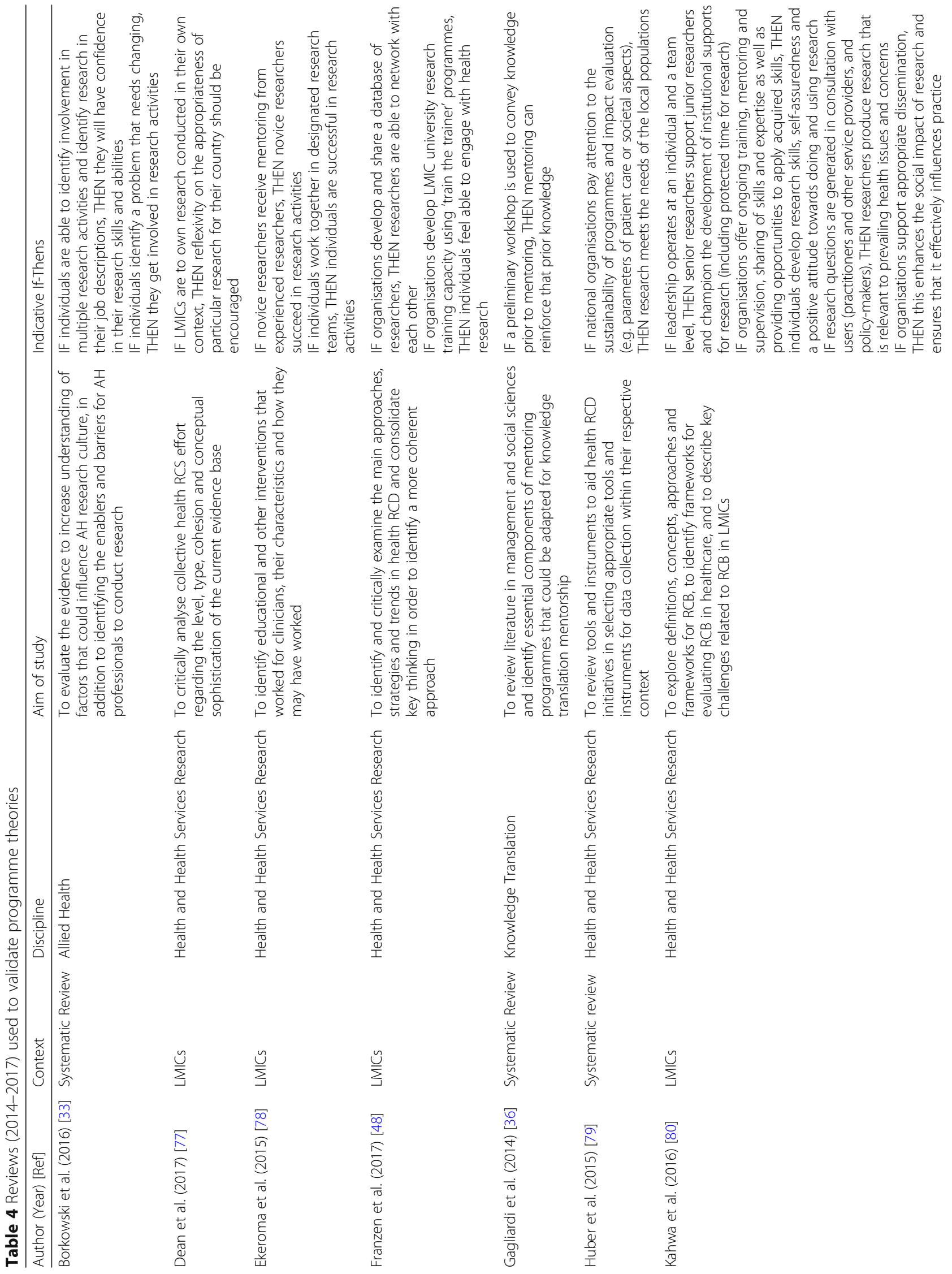




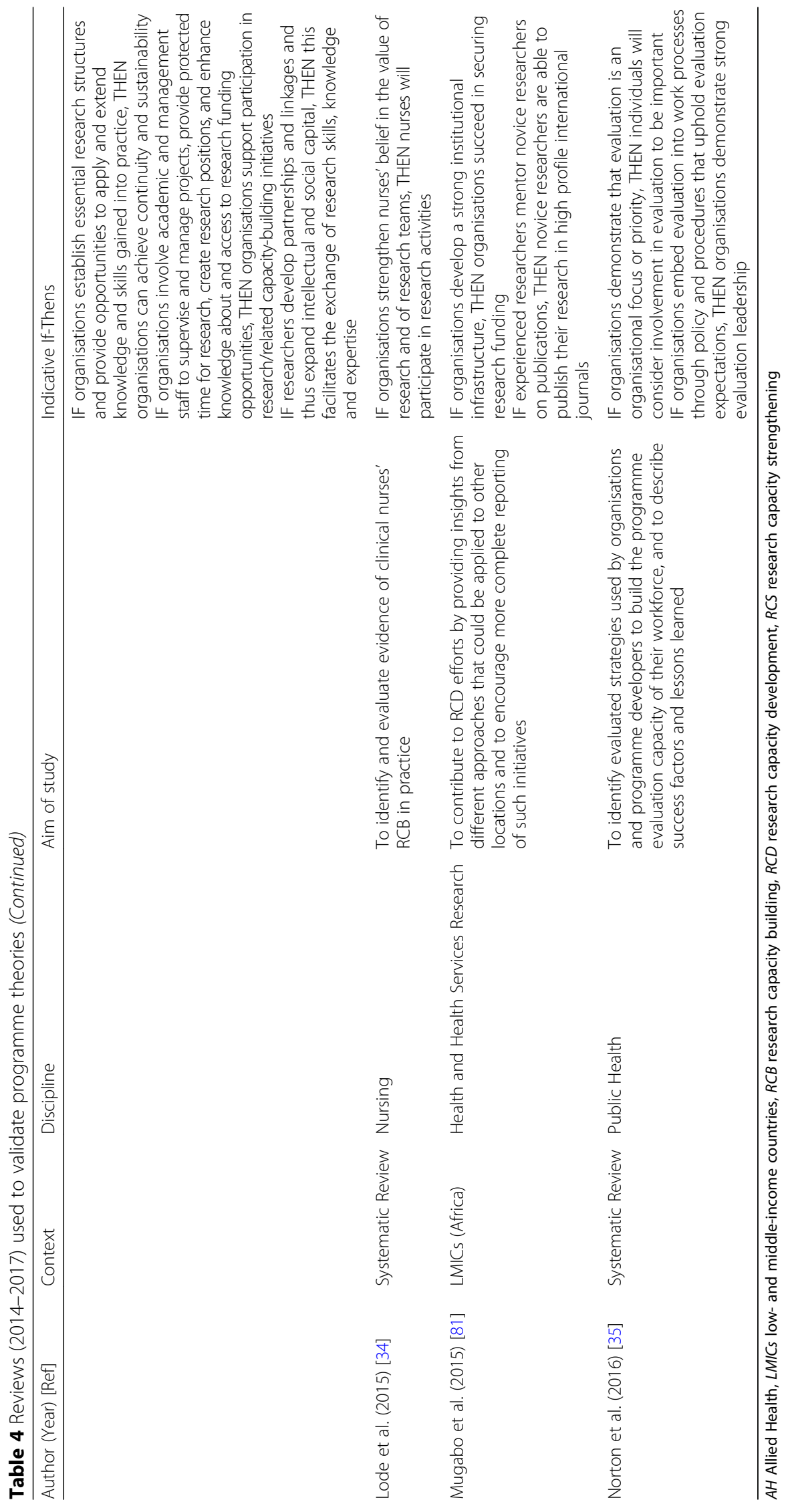


Table 5 Activities identified in reviews (2014-2017)

\begin{tabular}{|c|c|}
\hline Author (Year) [Ref] & $\begin{array}{l}\text { Research capacity development activities } \\
\text { included }\end{array}$ \\
\hline Borkowski et al. (2016) [33] & $\begin{array}{l}\text { Infrastructure, Leadership, Mentorship, } \\
\text { Training }\end{array}$ \\
\hline Dean et al. (2017) [77] & Infrastructure, Networks, Training \\
\hline Ekeroma et al. (2015) [78] & Mentoring, Networks, Training \\
\hline Franzen et al. (2017) [48] & Leadership, Mentoring, Networks, Training \\
\hline Gagliardi et al. (2014) [36] & Mentoring \\
\hline Huber et al. (2015) [79] & Infrastructure \\
\hline Kahwa et al. (2016) [80] & $\begin{array}{l}\text { Funding, Infrastructure, Leadership, Mentoring, } \\
\text { Networks, Training }\end{array}$ \\
\hline Lode et al. (2015) [34] & Funding, Leadership, Networks, Training \\
\hline Mugabo et al. (2015) [81] & Infrastructure, Mentoring, Training \\
\hline Norton et al. (2016) [35] & Infrastructure, Leadership, Training \\
\hline
\end{tabular}

academic engagement in health systems. Therefore, for example, the 'more than sum of parts' includes valuing and recognising as an asset, the contribution that each individual brings to the partnership, enabling discussion and joint thinking, access to different networks, and dividing workload across disciplines. This promotes respectful and meaningful discussion that can lead to trust and on-going dialogue and can be underlying mechanisms throughout diverse interventions, for example, in priority-setting, grant writing groups, communities of practice, knowledge transfer partnerships and doctoral training networks. Additionally, the resulting outcome provides a new context that can activate other mechanisms, for example, 'coproduction of research' and 'learning by doing'. Thus, the outcome of one mechanism can produce the context to stimulate mechanisms in others. Reviewing the programme theories in Table 3 reveals that they form chains of 'context $(\mathrm{C})$ - mechanism $(\mathrm{M})$ - outcome $(\mathrm{O})$ ', where the outcome of one part of the chain shapes a subsequent context within which to stimulate the mechanism in others in the RCD programme. Similarly, 'liberating talents', 'learning by doing' and 'releasing resources' form chains, which may be usefully harnessed when developing fellowship and secondment interventions, for example.

A major finding from the qualitative synthesis and analysis was that many activities fulfil an emblematic (symbolic) role in signalling the importance of RCD within the organisations, networks or teams. Therefore, for example, the 'protected time' or 'buy out from other responsibilities' interventions initially seem to serve an instrumental role in freeing staff from their other duties in order to participate in research. On closer examination, however, it is clear that such activities are equally important in demonstrating to staff within an organisation that research is important and should be considered an organisational/system priority. Similarly, the development of small funding schemes in research networks or organisations, engagement in writing workshops and the promulgation of mentorship schemes assume significance beyond their monetary value in signalling that research is valued and hence an activity in which it is legitimate for staff to engage. A common trigger for all mechanisms is 'making a difference', which can stimulate motivation in stakeholders across the RCD programme, and can be seen as a community building exercise.

\section{Validation and refreshment of programme theories}

Citation searches for the 10 citation pearls in December 2017 identified a further 10 systematic reviews published between 2014 and 2017 that met our original inclusion criteria (Table 4). Collectively, these covered all the RCD activities (Box 1) and reflected the diversity of contexts identified in the original dataset (e.g. high- $(n=5)$ and low- and middle-income countries $(n=5)$; allied health [33], nursing [34], public health [35], and health and health services research $(n=6)$, etc.). A new addition was in the emerging field of knowledge translation [36]. Again, the papers represented all the identified activities of RCD (Box 1), yet, since they were all reviews, they were more likely to describe multiple activities than the original dataset (Table 5).

\section{Box 1 Activities undertaken in research capacity development (RCD) (from Re:CAP [10])}

1. Prioritisation: Developing research priorities from consensus views of informed participants.

2. Mentoring: where an experienced, highly regarded person (the mentor) guides another individual (the mentee) in the development and examination of their own ideas, learning, and personal and professional development.

3. Leadership: the process of influencing group activities towards the achievement of RCD goals.

4. Research facilitators: individuals whose role is explicitly to promote and enable the conduct of a research by those with limited research experience.

5. Training: interventions that aim to increase skills and knowledge.

6. Funding to develop RCD including bursaries and fellowships.

7. Networks and collaborations: structures and functions that support people to work together to improve knowledge transfer, innovation, a research process or an output.

8. Infrastructure: a range of activities used to enhance support of RCD. 
The identified reviews confirmed the original programme theory, adding a nuanced understanding of many RCD activities. However, the new dataset did not identify any new strands of programme theory, possibly indicating that theoretical saturation had been reached. Evaluation, debatably an RCD activity in its own right, emerged as increasingly prominent in the recent literature. However, evaluation was considered to be a subtext to all the other activities and was not included as an additional activity. Future conceptual models should ensure that they feature evaluation, albeit to be handled differently from other activities.

\section{Discussion}

Many governments and global partnerships invest considerable funds to support RCD in healthcare, and it is a moral and ethical imperative to develop, shape and evaluate such activity [37] in order to plan and attain the desired effect, and to justify continued funding [16]. This unique realist synthesis of the conceptual literature on RCD has uncovered mechanisms that operate beneath such activities which, we suggest, can function across and within different structural levels with an emphasis of meaningful societal impact. We suggest that the programme theories developed here might help to plan and demonstrate cohesion and alignment across structural levels. Several authors recognise that RCD activities need to take place concurrently at a number of different levels $[1,8,13]$, with many calling for a 'whole systems' approach to $\operatorname{RCD}[3,19]$. We propose that the programme theories developed here could act as a guide for application across the diverse individual, organisational and network levels in order to promote synergy and ensure RCD activities 'pull' in the same direction.

Social change is the ultimate outcome for RCD [8]. The programme theories presented here, particularly those described as 'symbolic', can provide visible mechanisms of how RCD might influence culture, leadership and motivation. Many of these mechanisms have foundations in theories of social change and social capital, and provide an explanation of how interventions that adopt such an approach can engender a research culture within organisations and networks, and reciprocity and leadership in individuals.

Our programme theories resonate with others that explain how research activity can promote impact in healthcare organisations and communities [38, 39, 40]. Whilst training in research traditionally includes research methods, data collection and analysis skills, the co-production programme theory suggests diverse skills of cross boundary working, negotiation and creative practices in knowledge production [39]. Researchers using a co-productive approach are more likely to align research with stakeholder and organisational objectives [1] and form dynamic partnerships using assets from different organisations and networks [38] to make a difference.

Under what circumstances are RCD interventions most likely to achieve their intended effect? Our analysis has identified several principal components from a theoretical perspective:

1) RCD interventions may act as a catalyst for releasing potential research energies from within individuals and organisations. This is most clearly seen in the programme theories that relate to 'Exceeding the sum of the parts (PT1)', 'Liberating the talents (PT3)' and 'Releasing resources (PT4)'. The implication is that, without such triggers, the organisation and individuals remain essentially inert or slow moving with regard to their engagement in research activities.

2) RCDs must meet criteria for observability, meaning that current and potential participants must be able to perceive potential and actual benefits from their involvement. This is most clearly seen in the collective programme theories labelled as symbolic (or emblematic), i.e. 'Feeling that you are making a difference (PT6)', 'Modelling positive behaviours (PT7)' and 'Signalling importance (PT8)'. However, it is additionally present within the 'Learning by doing (PT2)' programme theory, where trainers and trainees receive almost synchronous confirmation of personal growth and skills acquisition.

3) RCDs must secure the engagement and commitment of their stakeholders and beneficiaries. Such commitment may be overtly signalled through explicit strategies or statements on research, through the celebration of achievements and through the provision of protected time from the demands of competing activities ('Signalling importance PT8). It can also be secured by cocreation opportunities through 'Releasing resources (PT4)' and 'Coproducing knowledge (PT5)' with added opportunities for 'Feeling that you are making a difference (PT5)'.

4) Possible linkages and $\mathrm{C}-\mathrm{M}-\mathrm{O}$ chains are emerging, where the outcome of one mechanism stimulates another within the programme architecture, and can act as leverage within it. For example, the mechanisms that are symbolic (PT6-8) may nurture a research culture that acts as a backdrop to other activities (P1-7). Variation in this cultural backdrop can be conceived as the effect of a dimmer switch [41] by which the range of outcomes of the symbolic mechanisms have correspondingly greater or lesser influence on associated RCD activities. The more evident a 
research culture, the more assets/resources that culture is able to bring to the RCD architecture and, correspondingly, the more power released within the dimmer switch to stimulate a range of mechanisms across the programme.

Thus, the findings from this realist synthesis of the conceptual RCD literature offer a starting point for constructing a RCD framework shaped by these programme theories. Future work should include exploration, elaboration and iterative refinement of these programme theories through exploration with other theory (some suggestions are included in Table 3), and testing against empirical data from intervention studies $[42,43]$.

\section{Strengths}

We have taken a first step in developing components of an overarching theory to determine what works for whom to accomplish 'more research done well'. Searches were conducted across a wide range of databases and were supplemented by exhaustive reference checking and citation tracking. The 36 conceptual and theoretical papers we identified are derived from diverse settings and describe RCD activities at multiple levels, strengthening confidence in the identification of candidate mechanisms. All major RCD activities are identifiable in both the included set of papers and in the validation set of recent reviews with which we tested our initial findings. Our realist-based approach offers an opportunity for a more nuanced understanding of how interventions might work and, indeed, in understanding circumstances in which they may not, which goes beyond the mere presence or absence of a specific intervention, a serendipitous bundle of collective interventions or a tailored package of synergistic initiatives.

\section{Limitations}

The systematic mapping process was subject to time and resource constraints and was primarily conducted by one investigator. Validation of a $20 \%$ sample was performed by the other two investigators to sensitise team members to the characteristics of the evidence base. The team reached a consensus on what should be recognised as constituting a model, theory or framework but did not distinguish between these three contested terms. The articles studied for the presence of programme theory were purposively sampled from a wide range of candidate studies and were selected to represent diverse settings and contexts and because of the perceived richness of their data.

Our preliminary findings have already been shared with a group of nine National Health Service organisations who meet to promote organisational development in RCD, called ACORN (Addressing Capacity in Organisations to do Research Network) [44]. Individuals were able to comment on the extent to which these programme theories fit with their practical experience of RCD.

Potential implications, based upon our theoretical frameworks, for those planning RCD at different contextual levels are given in Box 2.

\section{Conclusions}

Realist evaluation approaches are increasingly common when evaluating specific interventions in RCD [45, 46, 47]. Other authors are further using innovative literature review methods in order to explore development strategies for RCD [48]. We believe that this is the first time that the innovative approach of realist synthesis has been applied to conceptual papers on RCD in order to isolate the underpinning RCD programme theories. The value of this approach is in drilling down beneath the activities of a programme to identify the mechanisms that are deployed therein.

This review found that, collectively and individually, RCDs engage with multiple defined programme theories to achieve their potential impact. Such programme theories have a role in developing new RCD interventions, in modifying existing initiatives, and in creating a comprehensive evaluation framework against which to measure achievements. We have been able to tentatively explore how C-M-O might link to develop 'trigger' chains and speculate how symbolic mechanisms may link to interventional ones. This needs to be explored further within intervention studies. Our initial work requires further development to extend the analysis and thus cover a full range of RCD interventions, mapping both activities and evaluation measures by intervention, stage in the development lifecycle, and by programme theory.

Our investigation represents an overt attempt to capitalise on the utility of realist synthesis for the specific tasks of theory generation and subsequent exploration of potential mechanisms. Gough et al. [28] characterise the potential contributions of synthesis in general in terms of generating, exploring and testing (G-E-T) of theory. A subsequent stage of this project is therefore to test these emergent concepts with reference to empirical studies, either relating to research capacity as a composite activity or to the individual interventions by which we characterise research capacity activities. Planned outcomes from the subsequent stages of this project include identifying which mechanisms are associated with specific types of intervention and the development of an evaluation framework with which to assess the achievements of general RCD programmes and their constituent interventions.

Our novel evidence-based model identified 36 conceptually rich papers relevant to RCD. Although we acknowledge that other papers hold the potential to inform our 


\section{Box 2 Potential implications when planning RCD}

\section{Funding bodies}

- Develop research priority-setting mechanisms to release resources to fund research that can 'make a difference'. Priorities should be agreed between stakeholders to co-produce knowledge that will have an impact on health and wealth.

- Develop funding opportunities to support 'learning by doing' opportunities for individuals, to compliment more formal research training.

- Fund career pathways and liberate talents through actively seeking individuals with potential, and fund coaching and mentorship to maximise this.

- Fund appointments between healthcare and academic organisations to support partnerships that exceeds sum of parts and coproduction of research, and provide positive role models.

- Develop funding calls to a release resource and signal importance of research activity within healthcare organisations. For example, through a matched funding model in large research programmes, and protected time agreed with managers in 'learning by doing' opportunities.

- Fund novel methods of research dissemination that promote action in clinical and healthcare practice so that the research findings can make a difference, and signal importance of implementing research knowledge into practice.

\section{Healthcare organisations}

- Signal the importance of research activity within the organisation through job descriptions, mission statements, training and R\&D strategies. Support business plans in order to release resources that will include protected research time and ring-fenced research resources.

- Recognise and celebrate positive research behaviours in clinical academic staff, managers and services through award schemes and communication channels.

- Enable mentoring and coaching schemes to be undertaken in their organisation in order to release potential talent and support learning by doing activities.

- Seek and support 'learning by doing' programmes as well as more traditional research training opportunities.

- Develop a needs and assets register to recognise and liberate talent.

- Develop a sense of ownership and commitment to research activity, through co-creation of research ideas, observable instances of quick wins and impact success stories, to demonstrate research that makes a difference.

- Work proactively in partnership with other organisations, networks and academic institutions to maximise synergies, coproduction, 'learning by doing' opportunities.

Individuals:

- Recognise the personal, organisational and long-term benefits from their own involvement in research to make a difference and to demonstrate positive research behaviours.

- Seek and use leaning by doing opportunities.

- Support release of their own talents, and that of others around them. Be both a mentor and a mentee. Use coaching opportunities to release their own potential.

- Work with managers to negotiate protected time to signal importance of research alongside practice.

- Develop skills to support coproduction of research, and plan research activity that has an impact on practice to make a difference.

- Are able to develop skills and knowledge through practical involvement in research activities, including alignment to organisational objectives. 
theory development, particularly in relation to the characteristics of individual interventions, we believe that we have identified the more common and significant programme theories that relate to $\mathrm{RCD}$. We anticipate that further exploration will reveal a point of theoretical saturation and may help in identifying some of the nuances or, indeed, disconfirming cases, associated with specific RCD initiatives. Ultimately, we hope that the conceptual framework presented in this paper will contribute to the demonstration of long-term outcomes in health, wealth and knowledge as commissioners and service providers work together to increase $\mathrm{RCD}$.

\section{Additional file}

Additional file 1: The 36 studies describing conceptual models, frameworks or theory for research capacity development. (DOCX 35 kb)

\section{Abbreviations}

KM: knowledge mobilisation; RCD: research capacity development; Re:CAP: Research Capacity - A scoping review to identify the evidence-base for Research Capacity development in health and social care

\section{Funding}

The authors of this paper were funded and supported by the National Institute for Health Research (NIHR) Collaboration for Leadership in Applied Health Research and Care Yorkshire and Humber (NIHR CLAHRC YH) (www.clahrc-yh.nihr.ac.uk). The views and opinions expressed are those of the authors, and not necessarily those of the National Health Service, the NIHR or the Department of Health.

\section{Availability of data and materials}

The datasets used and/or analysed during the current study are available from the corresponding author on reasonable request.

\section{Authors' contributions}

$J C$ and $A B$ conceived the study. PG conducted the subject searches and pursued the citation pearls. All three authors undertook study selection. JC and $A B$ extracted data from included studies. All authors participated in the analysis and the interpretation of review findings. All authors read and approved the final manuscript.

\section{Ethics approval and consent to participate}

Not relevant, this is a literature review with no primary research data or data from human subjects.

\section{Consent for publication}

No individual person's data in any form.

\section{Competing interests}

The authors declare that they have no competing interests.

\section{Publisher's Note}

Springer Nature remains neutral with regard to jurisdictional claims in published maps and institutional affiliations.

\section{Author details}

${ }^{1}$ NIHR CLAHRC Yorkshire \& Humber, Research Capacity and Engagement Programme Management, 11 Broomfield Road, Sheffield S10 2SE, United Kingdom. ${ }^{2}$ Department of Public Health and Pediatrics, University of Turin, Turin, Italy. ${ }^{3}$ School of Health and Related Research, University of Sheffield, Regent Court, 30 Regent Street, Sheffield S1 4DA, United Kingdom.
Received: 7 April 2018 Accepted: 17 August 2018

Published online: 21 September 2018

\section{References}

1. Lansang MA, Dennis R. Building capacity in health research in the developing world. Bull World Health Organ. 2004;82(10):764-70

2. Gorard S, Taylor C. Welcome to Building Research Capacity. ESRC Teaching and Learning Research Programme Research Capacity Building Network. 2002.

3. Whitworth A, Haining S, Stringer H. Enhancing research capacity across healthcare and higher education sectors: development and evaluation of an integrated model. BMC Health Serv Res. 2012;12:287.

4. Albert E, Mickan S. Closing the gap and widening the scope. New directions for research capacity building in primary health care. Aust Fam Physician. 2003;32(12):1038-40. 1043

5. Mahmood S, Hort K, Ahmed S, Salam M, Cravioto A. Strategies for capacity building for health research in Bangladesh: role of core funding and a common monitoring and evaluation framework. Health Res Policy Syst. $2011 \cdot 9 \cdot 31$

6. Jenerette CM, Funk M, Ruff C, Grey M, Adderley-Kelly B, McCorkle R. Models of inter-institutional collaboration to build research capacity for reducing health disparities. Nurs Outlook. 2008;56(1):16-24.

7. Department of Health. Best Research for Best Health: A New National Health Research Strategy. London: Department of Health; 2006.

8. Condell SL, Begley C. Capacity building: a concept analysis of the term applied to research. Int J Nurs Pract. 2007;13(5):268-75

9. Cooke J. A framework to evaluate research capacity building in health care. BMC Fam Pract. 2005;6:44.

10. Cooke J, Booth A, Nancarrow S, Wilkinson A, Askew D. Re:cap - Identifying the Evidence-base for research capacity development in health and social care. A scoping review of the literature. Sheffield: Trent Research \& Development Support Unit, University of Sheffield; 2006.

11. Sambunjak D, Straus SE, Marušić A. Mentoring in academic medicine: a systematic review. Jama. 2006;296(9):1103-15.

12. Cooke J, Ariss S, Smith C, Read J. On-going collaborative priority-setting for research activity: a method of capacity building to reduce the researchpractice translational gap. Health Res Policy Syst. 2015;13:25.

13. Levine R, Russ-Eft D, Burling A, Stephens J, Downey J. Evaluating health services research capacity building programs: implications for health services and human resource development. Eval Program Plann. 2013;37:1-11.

14. Macfarlane F, Shaw S, Greenhalgh T, Carter YH. General practices as emergent research organizations: a qualitative study into organizational development. Fam Pract. 2005;22(3):298-304.

15. Nuyens $Y$. Setting priorities for health research: lessons from low-and middle-income countries. Bull World Health Organ. 2007;85(4):319-21.

16. Gadsby EW. Research capacity strengthening: donor approaches to improving and assessing its impact in low- and middle-income countries. Int J Health Plann Manag. 2011;26(1):89-106.

17. Willis $C$, Riley $B$, Lewis $M$, Stockton L, Yessis J. Guidance for organisational strategy on knowledge to action from conceptual frameworks and practice. Evid Policy. 2017;13(2):317-41.

18. Del Mar C, Askew D. Building family/general practice research capacity. Ann Fam Med. 2004;2(Suppl 2):S35-40.

19. Farmer E, Weston K. A conceptual model for capacity building in Australian primary health care research. Aust Fam Physician. 2002;31(12):1139-42.

20. Johnson K, Kennedy SB, Harris AO, Lincoln A, Neace W, Collins D. Strengthening the HIV/AIDS service delivery system in Liberia: an international research capacity-building strategy. J Eval Clin Pract. 2005;11(3):257-73.

21. Breen C, Jaganyi J, Van Wilgen B, Van Wyk E. Research projects and capacity building. Water SA. 2004;30(4):429-34

22. Pawson R, Greenhalgh T, Harvey G, Walshe K. Realist review - a new method of systematic review designed for complex policy interventions. J Health Serv Res Policy. 2016;10(1_Suppl):21-34.

23. Rycroft-Malone J, McCormack B, Hutchinson AM, DeCorby K, Bucknall TK, Kent B, Schultz A, Snelgrove-Clarke E, Stetler CB, Titler M, Wallin L, Wilson V. Realist synthesis: illustrating the method for implementation research. Implement Sci 2012;7:33

24. Kavanagh J, Trouton A, Oakley A, Powell C. A scoping review of the evidence for incentive schemes to encourage positive health and other social behaviours in young people. EPPI-Centre, Social Science Research Unit, Institute of Education, University of London: London; 2005. 
25. Booth A, Carroll C. Systematic searching for theory to inform systematic reviews: is it feasible? Is it desirable? Health Inf Libr J. 2015;32(3):220-35.

26. Jones A, Burgess TA, Farmer EA, Fuller J, Stocks NP, Taylor JE, Waters RL. Building research capacity. An exploratory model of GPs' training needs and barriers to research involvement. Aust Fam Physician. 2003;32(11):957-60.

27. North American Primary Care Research Group Committee on Building Research Capacity. What does it mean to build research capacity? Fam Med. 2002;34(9):678-84

28. Gough D, Thomas J, Oliver S. Clarifying differences between review designs and methods. Syst Rev. 2012;1:28.

29. Pawson R. Evidence-based policy: the promise of realist synthesis. Evaluation. 2002;8(3):340-58.

30. Booth A, Sutton A, Papaioannou D. Systematic Approaches to a Successful Literature Review. 2nd edn. London: Sage; 2016.

31. Coen SE, Bottorff JL, Johnson JL, Ratner PA. A relational conceptual framework for multidisciplinary health research Centre infrastructure. Health Res Policy Syst. 2010;8:29.

32. Patton M. Qualitative Research and Evaluation Methods. 3rd ed. Thousand Oaks: Sage Publications; 2002

33. Borkowski D, McKinstry C, Cotchett M, Williams C, Haines T. Research culture in allied health: a systematic review. Aust J Prim Health. 2016;22(4):294-303.

34. Lode K, Sørensen EE, Salmela S, Holm AL, Severinsson E. Clinical nurses' research capacity building in practice-a systematic review. Open J Nurs. 2015;5(07):664.

35. Norton S, Milat A, Edwards B, Giffin M. Narrative review of strategies by organizations for building evaluation capacity. Eval Program Plann. 2016;58: 1-19. https://doi.org/10.1016/j.evalprogplan.2016.04.004

36. Gagliardi AR, Webster F, Perrier L, Bell M, Straus S. Exploring mentorship as a strategy to build capacity for knowledge translation research and practice: a scoping systematic review. Implement Sci. 2014;9:122.

37. Faden RR, Kass NE, Goodman SN, Pronovost P, Tunis S, Beauchamp TL. An ethics framework for a learning health care system: a departure from traditional research ethics and clinical ethics. Hast Cent Rep. 2013;43(s1): S16-27. https://doi.org/10.1002/hast.134.

38. Greenhalgh T, Jackson C, Shaw S, Janamian T. Achieving research impact through co-creation in community-based health services: literature review and case study. Milbank Q. 2016;94(2):392-429.

39. Rycroft-Malone J, Burton CR, Bucknall T, Graham ID, Hutchinson AM, Stacey D. Collaboration and co-production of knowledge in healthcare: opportunities and challenges. Int J Health Policy Manag. 2016;5(4):221.

40. Boaz A, Hanney S, Jones T, Soper B. Does the engagement of clinicians and organisations in research improve healthcare performance: a three-stage review. BMJ Open. 2015;5(12):e009415.

41. Dalkin SM, Greenhalgh J, Jones D, Cunningham B, Lhussier M. What's in a mechanism? Development of a key concept in realist evaluation. Implement Sci. 2015;10(1):49.

42. Wong G, Greenhalgh T, Westhorp G, Pawson R. Realist methods in medical education research: what are they and what can they contribute? Med Educ. 2012;46(1):89-96.

43. Van Belle $S$, van de Pas R, Marchal B. Towards an agenda for implementation science in global health: there is nothing more practical than good (social science) theories. BMJ Glob Health. 2017;2(2):e000181.

44. Gee M, Cooke J. How do NHS organisations plan research capacity development? Strategies, strengths, and opportunities for improvement. BMC Health Serv Res. 2018;18(1):198.

45. Marjanovic S, Cochrane G, Robin E, Sewankambo N, Ezeh A, Nyirenda M, Bonfoh B, Rweyemamu M, Chataway J. Evaluating a complex research capacity-building intervention: reflections on an evaluation of the African Institutions Initiative. Evaluation. 2017;23(1):80-101.

46. Wenke RJ, Ward EC, Hickman I, Hulcombe J, Phillips R, Mickan S. Allied health research positions: a qualitative evaluation of their impact. Health Res Policy Syst. 2017;15(1):6.

47. Mickan S, Wenke R, Weir K, Bialocerkowski A, Noble C. Strategies for research engagement of clinicians in allied health (STRETCH): a mixed methods research protocol. BMJ Open. 2017;7(9):e014876. https://doi.org/10. 1136/bmjopen-2016-014876.

48. Franzen SR, Chandler C, Lang T. Health research capacity development in low and middle income countries: reality or rhetoric? A systematic metanarrative review of the qualitative literature. BMJ Open. 2017;7(1):e012332.

49. Conn VS, Porter RT, McDaniel RW, Rantz MJ, Maas ML. Building research productivity in an academic setting. Nurs Outlook. 2005;53(5):224-31.
50. Cooke A, Green B. Developing the research capacity of departments of nursing and midwifery based in higher education: a review of the literature. J Adv Nurs. 2000;32(1):57-65.

51. Edwards N, Webber J, Mill J, Kahwa E, Roelofs S. Building capacity for nurseled research. Int Nurs Rev. 2009;56(1):88-94.

52. Fenton E, Harvey J, Griffiths F, Wild A, Sturt J. Reflections from organization science on the development of primary health care research networks. Fam Pract. 2001;18(5):540-4.

53. Fenton E, Harvey J, Sturt J. Evaluating primary care research networks. Health Serv Manag Res. 2007;20(3):162-73.

54. Fitzgerald M, Milberger P, Tomlinson PS, Peden-mcalpine C, Meiers SJ, Sherman S. Clinical nurse specialist participation on a collaborative research project: barriers and benefits. Clin Nurse Spec. 2003;17(1):44-9.

55. Golenko X, Pager S, Holden L. A thematic analysis of the role of the organisation in building allied health research capacity: a senior managers' perspective. BMC Health Serv Res. 2012;12:276.

56. Green B, Segrott J, Priest H, Rout A, Mclvor M, Douglas J, Flood Y, Morris S, Rushton C. Research capacity for everyone? A case study of two academic nursing schools' capacity building strategies. J Res Nurs. 2007;12(3):247-65.

57. Nchinda TC. Research capacity strengthening in the south. Soc Sci Med. 2002;54(11):1699-711.

58. O'Byrne L, Smith S. Models to enhance research capacity and capability in clinical nurses: a narrative review. J Clin Nurs. 2011;20(9-10):1365-71.

59. Pickstone C, Nancarrow SA, Cooke JM, Vernon W, Mountain GA, Boyce RA, Campbell J. Building research capacity in the allied health professions. Evid Policy. 2008;4(1):53-68.

60. Priest H, Segrott J, Green B, Rout A. Harnessing collaboration to build nursing research capacity: a research team journey. Nurse Educ Today. 2007;27(6):577-87.

61. Raghunath $A$, Innes $A$. The case for multidisciplinary research in primary care. Prim Health Care Rese Dev. 2004;5(3):264-73.

62. Ried K, Fuller J. Building a culture of research dissemination in primary health care: the south Australian experience of supporting the novice researcher. Aust Health Rev. 2005;29(1):6-11.

63. Ried K, Farmer EA, Weston KM. Setting directions for capacity building in primary health care: a survey of a research network. BMC Fam Pract. 2006;7:8.

64. Ried K, Farmer EA, Weston KM. Bursaries, writing grants and fellowships: a strategy to develop research capacity in primary health care. BMC Fam Pract. 2007:8:19.

65. Sarre G, Cooke J. Developing indicators for measuring research capacity development in primary care organizations: a consensus approach using a nominal group technique. Health Soc Care Community. 2009;17(3):244-53.

66. Segrott J, Mclvor M, Green B. Challenges and strategies in developing nursing research capacity: a review of the literature. Int J Nurs Stud. 2006;43(5):637-51.

67. Stephens J, Levine R, Burling A, Russ-Eft D. An Organizational Guide to Building Health Services Research Capacity, Final Report (AHRQ Publication No 11 (12)0095-EF). Rockville: Agency for Healthcare Research and Quality; 2011.

68. van Weel C, Rosser WW. Improving health care globally: a critical review of the necessity of family medicine research and recommendations to build research capacity. Ann Fam Med. 2004;2(Suppl 2):S5-16.

69. Lave J, Wenger E. Situated Learning: Legitimate Peripheral Participation. New York: Cambridge University Press; 1991.

70. Green B, Segrott J, Hewitt J. Developing nursing and midwifery research capacity in a university department: case study. J Adv Nurs. 2006;56(3):302-13.

71. Beresford P. Developing the theoretical basis for service user/survivorled research and equal involvement in research. Epidemiol Psychiatr Sci. 2005;14(1):4-9.

72. Boyle D, Harris M. The Challenges of Co-Production: How Equal Partnerships between Professionals and the Public are Crucial to Improving Public Services. London: Nesta; 2009.

73. Heaton J, Day J, Britten N. Inside the "black box" of a knowledge translation program in applied health research. Qual Health Res. 2015;25(11):1477-91.

74. Cole DC, Aslanyan G, Dunn A, Boyd A, Bates I. Dilemmas of evaluation: health research capacity initiatives. Bull World Health Organ. 2014;92(12): 920-1.

75. Ried K, Montgomery BD, Stocks NP, Farmer EA. General practice research training: impact of the Australian Registrar Research Workshop on research skills, confidence, interest and involvement of participants, 2002-2006. Fam Pract. 2008;25(2):119-26. https://doi.org/10.1093/fampra/cmn010. 
76. Perry L, Grange A, Heyman B, Noble P. Stakeholders' perceptions of a research capacity development project for nurses, midwives and allied health professionals. J Nurs Manag. 2008;16(3):315-26.

77. Dean L, Gregorius S, Bates I, Pulford J. Advancing the science of health research capacity strengthening in low-income and middle-income countries: a scoping review of the published literature, 2000-2016. BMJ Open. 2017;7(12):e018718.

78. Ekeroma A, Kenealy T, Shulruf B, Hill A. Educational and wider interventions that increase research activity and capacity of clinicians in low to middle income countries: a systematic review and narrative synthesis. J Res Dev. 2015:3(1):1000120.

79. Huber J, Bauer D, Hoelscher M, Kapungu J, Kroidl A, Lennemann T, Maganga L, Opitz O, Salehe O, Sigauke A, et al. Evaluation of health research capacity strengthening trainings on individual level: validation of a questionnaire. J Eval Clin Pract. 2014:20(4):390-5

80. Kahwa E, Edwards N, Mortley N. Research capacity building: a literature review and the theoretical framework. In: Edwards N, Kaseje D, Kahwa $E_{i}$ editors. Building and evaluating research capacity in healthcare systems: case studies and innovative models. Ottawa: UCT Press; 2016. p. 1-260.

81. Mugabo L, Rouleau D, Odhiambo J, Nisingizwe MP, Amoroso C, Barebwanuwe P, Warugaba C, Habumugisha L, Hedt-Gauthier BL. Approaches and impact of non-academic research capacity strengthening training models in sub-Saharan Africa: a systematic review. Health Res Policy Syst. 2015;13:30

Ready to submit your research? Choose BMC and benefit from:

- fast, convenient online submission

- thorough peer review by experienced researchers in your field

- rapid publication on acceptance

- support for research data, including large and complex data types

- gold Open Access which fosters wider collaboration and increased citations

- maximum visibility for your research: over $100 \mathrm{M}$ website views per year

At BMC, research is always in progress.

Learn more biomedcentral.com/submissions 